\title{
Using Gene Expression to Improve the Power of Genome-Wide Association Analysis
}

\author{
Yen-Yi Ho ${ }^{a}$ Emily C. Baechler ${ }^{b}$ Ward Ortmann ${ }^{c}$ Timothy W. Behrens ${ }^{c}$ \\ Robert R. Graham ${ }^{\text {c Tushar R. Bhangale }}{ }^{d}$ Wei Pan ${ }^{a}$ \\ ${ }^{a}$ Division of Biostatistics and ${ }^{b}$ Department of Medicine, University of Minnesota, Minneapolis, Minn., and \\ Departments of ${ }^{\mathrm{C} H u m a n}$ Genetics and ${ }^{\mathrm{d} B i o i n f o r m a t i c s}$ and Computational Biology, Genentech, Inc., \\ San Francisco, Calif., USA
}

\section{Key Words}

$\mathrm{p}$ value weighting $\cdot$ Family-wise error rate $\cdot$ Statistical power . Integrative genomic analysis · SLE

\begin{abstract}
Background/Aims: Genome-wide association (GWA) studies have reported susceptible regions in the human genome for many common diseases and traits; however, these loci only explain a minority of trait heritability. To boost the power of a GWA study, substantial research endeavors have been focused on integrating other available genomic information in the analysis. Advances in high through-put technologies have generated a wealth of genomic data and made combining SNP and gene expression data become feasible. $\boldsymbol{R e}$ sults: In this paper, we propose a novel procedure to incorporate gene expression information into GWA analysis. This procedure utilizes weights constructed by gene expression measurements to adjust $p$ values from a GWA analysis. Results from simulation analyses indicate that the proposed procedures may achieve substantial power gains, while controlling family-wise type I error rates at the nominal level. To demonstrate the implementation of our proposed approach, we apply the weight adjustment procedure to a GWA study on serum interferon-regulated chemokine levels in systemic
\end{abstract}

lupus erythematosus patients. The study results can provide valuable insights for the functional interpretation of GWA signals. Availability: The R source code for implementing the proposed weighting procedure is available at http:// www.biostat.umn.edu/ yho/research.html.

(c) 2014 S. Karger AG, Basel

\section{Introduction}

Over the past few years, genome-wide association (GWA) studies have been successful in localizing and identifying genetic regions that are related to common human diseases [1]. But studies have shown that the amount of genetic variation explained by GWA findings for any given disease is often significantly less than the estimated heritability of the disease [2]. One possible reason for the missing heritability is that GWA studies are underpowered to detect genetic variants that possess small effects. Most common human diseases or traits have complex inheritance patterns with multiple underlying genes with small to moderate effects. Therefore, it requires a relatively large sample size for a GWA study to detect signals with such small effects. In order to boost the statistical power of a GWA study, an important direction

\section{KARGER}

E-Mail karger@karger.com

www.karger.com/hhe
(C) 2014 S. Karger AG, Basel

0001-5652/14/0782-0094\$39.50/0
Yen-Yi Ho

Division of Biostatistics, University of Minnesota

420 Delaware St. S.E., Mayo Building, MMC 303

Minneapolis, MN 55455 (USA)

E-Mail yho@umn.edu 
of recent research is to integrate available genomic information such as gene expression, SNP, copy number variation, transcription regulation, methylation, and protein abundance together in the analysis $[3,4]$. With the advances of high through-put technologies, integrating gene expression with SNP information has drawn much attention in the past decade [5-11]. A recent study incorporating gene expression information into gene association mapping in mice showed assuring results in identifying functional networks that explain phenotypic alterations [12].

Genovese et al. [13] introduced the idea of utilizing prior knowledge to weight $p$ values from a GWA study. Li et al. [14] adapted the $p$ value weighting idea and proposed using gene expression information to formally derive weights and to apply the derived weights to adjust the $p$ values from a GWA study. They specified weights as

$$
w_{i}=\sqrt{-\log _{10} p_{e Q T L}},
$$

where $p_{e Q T L}$ is the $\mathrm{p}$ value for association between a SNP marker and gene expression (these SNP markers have sometimes been referred to as eQTLs). Li et al. [14] demonstrated a power gain when incorporating information of association between SNP markers and gene expression profiles in their study. However, their approach did not utilize the information of association between gene expression profiles and phenotypic outcome of interest.

In this paper, we propose approaches that utilize both $\mathrm{SNP}$-gene expression as well as gene expression-phenotype associations and which are expected to achieve greater power gains than that of Li et al. [14] in some situations. A study-wise threshold for the weight-adjusted $\mathrm{p}$ values can then be used to determine genome-wide significance. In addition to the expected power gain, the weights calculated based on gene expression can provide useful information for prioritizing SNPs for further functional validation experiments.

To assess the performance of our proposed approach, we conducted simulation analyses under various scenarios to evaluate the family-wise type I error rates (FWERs) and statistical power. We also compared the performance of our proposed weighting approaches to that of $\mathrm{Li}$ et al. [14] in the simulation analysis.

We apply the proposed approach to a study related to lupus activity in systemic lupus erythematosus (SLE) patients. Through experimental data analysis, our primary focus was to demonstrate the implementation of the proposed weighting adjustment approach. SLE is a chronic, inflammatory, autoimmune disease that can cause damage to organs and tissues throughout the body. The exact

Using Gene Expression to Improve the

Power of GWA cause of SLE is unknown, but a combination of genetic and environmental factors is thought to trigger the disease. More than 45 genetic variants are known to be associated with SLE, and over half of them can be linked to the type I interferon (IFN) pathway [15]. The type I IFNs are a family of antiviral cytokines that are implicated in the pathogenesis of lupus, and IFN-inducible transcripts and proteins are candidate biomarkers for this disease [16-19]. We previously evaluated a panel of 3 IFN-inducible serum chemokines (IP-10, MCP-1, MIP-3 $\beta$ ) as predictors of lupus flare [20]. The identification of genetic variants associated with elevated chemokine levels could improve the treatment of SLE patients and may assist in identifying additional disease susceptibility loci.

The paper is structured as follows: in the next section, we describe the study population and the weighting approach in detail, followed by the simulation analysis. Then, the implementation of the proposed approach in the SLE GWA study is described, ending with a discussion and a final conclusion.

\section{Research Design and Methods}

\section{Phenotype and Study Population}

In this study, our primary outcome of interest is lupus activity in SLE patients. Three IFNs-regulated serum chemokines, i.e. CXCL10 (IP-10), CCL2 (MCP-1) and CCL19 (MIP-3 $\beta$ ), were measured using SearchLight (Pierce, Woburn, Mass., USA) chemiluminescence sandwich-based immunoassays. A single, normalized, composite chemokine score on a 100-point scale was calculated using the three chemokine measurements as described in Bauer et al. [20]. The chemokine score could serve as a biomarker for lupus activity and predict future disease flares in the patient cohort [20].

The data we used was collected from 309 SLE patients with consent from the Hopkins Lupus Cohort [21] enrolled through the Autoimmune Biomarkers Collaborative Network (ABCoN) described in Bauer et al. [20]. All patients were Caucasian, and the majority was female. They received treatments for lupus during the study period including hydroxychloroquine, cyclophosphamide, mycophenolate mofetil, azathioprine, methotrexate, chlorambucil, and oral prednisone.

\section{SNP Genotyping and Gene Expression Profiling}

Whole genome genotyping for 555,352 SNP markers was carried out using Illumina 550K SNP array version 1 (HJ550v1; Illumina Inc., San Diego, Calif., USA). Gene expression of 24,849 genes was measured using Illumina Human-6 Expression BeadChip; data files were analyzed with Illumina's GenomeStudio gene expression module to report quantile-normalized, backgroundcorrected gene expression signal levels.

\section{Method}

In this study, we propose using weights $[13,22,23]$ computed and based on gene expression measurements to adjust the $p$ values from GWA analysis. Roeder et al. [24] suggested using weight $w_{i}$ 
$>0$ to adjust $\mathrm{p}$ value $p_{i}$ and reject null hypothesis $H_{i}$ if the adjusted $\mathrm{p}$ value is smaller than the Bonferroni-corrected threshold $\alpha / m$, where $\alpha$ is the significance level and $m$ is the total number of hypotheses. Hence, the set of rejected hypotheses $\left(H_{i}\right)$ is defined as:

$$
i \in R=\left(i: \frac{p_{i}}{w_{i}} \leq \frac{\alpha}{m}\right) .
$$

Roeder and Wasserman [25] provided theoretical proof that the rejection set $R$ described above controls the FWER at level $\alpha$, as long as $w_{i}>0$ and $\bar{w}_{i}=1$.

Based on their theoretical results, we utilize the $\mathrm{p}$ value weighting approach to gain power in detecting signal, while controlling the FWER at the nominal level. After weight adjustment, SNP loci that strongly contribute to a phenotype-associated gene expression will have smaller $\mathrm{p}$ values. The weighting mechanism is as follows: we assign a weight $w_{i}^{*}$ to each SNP marker $i$, such that $w_{i}^{*}>0$ and the average of all weights $\bar{w}^{*}$ is 1 . The weight for a given SNP $\left(L_{i}\right)$ is a product of two parts: $w_{L_{\mathrm{i}} E_{j}}$ and $w_{E_{\mathrm{j}} P} \cdot w_{L_{\mathrm{i}} E_{\mathrm{j}}}$ indicates the effect of SNP locus $L_{i}$ on the $j$-th gene expression measurement $E_{j}$. The second term $w_{E_{i} P}$ describes whether gene expression measurement $E_{j}$ is associated with the phenotypic outcome $P$.

To ensure a correct control of the FWER, we use the model below to calculate $w_{L_{\mathrm{i}} E_{\mathrm{j}}}$ :

$$
E_{j}=\beta_{0}+\beta_{L_{\mathrm{i}} E_{j}} L_{i}+\gamma P+\varepsilon, \quad L_{i}=0,1,2,
$$

and

$$
w_{L_{i} E_{j}}=\left(\frac{\widehat{\beta_{L_{i} E_{j}}}}{S E\left(\widehat{\beta_{L_{i} E_{j}}}\right)}\right)^{2} .
$$

The estimate

$$
\widehat{\beta_{L_{i} E_{j}}}
$$

from the above regression model is equivalent to $\beta_{r}$ in the following model:

$$
r_{1}=\alpha+\beta_{r} r_{2}+\varepsilon_{3}
$$

where $r_{1}$ and $r_{2}$ are residuals from the following 2 models, respectively:

$$
\begin{aligned}
& E_{j}=\alpha_{0}+\alpha_{1} P+\varepsilon_{1}, \\
& L_{i}=\alpha_{3}+\alpha_{4} P+\varepsilon_{2} .
\end{aligned}
$$

In equation 1 , the reason for adjusting for $P$ is to remove the effect of $P$, in order to prevent the correlation between weight $w_{L_{i} E_{j}}$ and the GWA p value. In an extreme situation, for example, consider SNP marker $L_{i}$ is not associated with $E_{j}$, while $E_{j}$ and $P$ are highly correlated. The $\mathrm{p}$ value for $\beta_{L_{\mathrm{i}} E_{\mathrm{j}}}$ in the model

$$
E_{j}=\beta_{0}+\beta_{L_{\mathrm{i}} E_{j}} L_{i}+\varepsilon
$$

will be highly correlated with the GWA p value for $\beta$ in the GWA model

$$
P=\beta_{0}+\beta L_{i}+\varepsilon,
$$

due to the high correlation between $E_{j}$ and $P$. Hence, after adjusting for the effect of $P$ in equation 1, the $\mathrm{p}$ value for $\beta_{L_{i} E_{j}}$ will be less likely to be significant, yielding a derived weight not highly correlated with the GWA p value.
Similarly, we adjust for $L_{i}$ when calculating weight $w_{E_{j} P}$ using the model

$$
P=\beta_{0}+\beta_{E_{j} P} E_{j}+\gamma L_{i}+\varepsilon
$$

and assign

$$
w_{E_{j} P}=\left(\frac{\widehat{\beta_{E_{j} P}}}{S E\left(\widehat{\beta_{E_{j} P}}\right)}\right)^{2} .
$$

Then, the product of the 2 weights is used to describe the effect of SNP locus $L_{i}$ on $P$ through $E_{j}$. The useful benefit of taking the product of 2 weights is that if either $w_{L_{i} E_{j}}$ or $w_{E_{\mathrm{j}} P}$ is zero, then the resulting product will be zero. On the other hand, if both $w_{L_{i} E_{j}}$ and $w_{E_{i} P}$ are reasonably large, then taking the product of the two parts will result in an amplified overall weight. A crude weight for SNP locus $L_{i}$ is determined by the maximum of the products among all gene expression measurements:

$$
w_{M P_{\mathrm{i}}}=\max _{j}\left(w_{L_{\mathrm{i}} E_{\mathrm{j}}} \times w_{E_{\mathrm{j}} P}\right) .
$$

Finally, we divide crude weights $w_{M P}$ by their average $w_{\bar{M} P}$, so that $\bar{w}^{*}$ is 1 , as required by Roeder and Wasserman [25]:

$$
w_{M P_{i}}^{*}=\frac{w_{M P_{i}}}{w_{M P_{i}}} .
$$

With $w_{M P_{i}}^{*}$, the adjusted $\mathrm{p}$ value for the $i$-th SNP can then be calculated as:

$$
\text { adjusted } \mathrm{p} \text { value for SNP } i=\frac{\mathrm{p} \text { value of SNP } i \text { reported from GWAS }}{w_{M P_{i}}^{*}} .
$$

In the weighting approach described above, we assume that the effect of the SNP genotype on the gene expression is linear. More generally, we may code 2 dummy variables to indicate $L_{i}=1$ versus 0 , and $L_{i}=2$ versus 0 , respectively, then the $\chi^{2}$ statistic derived from the likelihood ratio test can be used in replacement of the squared $\mathrm{t}$ statistic. Furthermore, in the case-control study design, a similar approach can be applied by deriving the weights through logistic regression.

Because the weights are constructed as the products of 2 parts, to ensure both the associations between $L_{i}$ and $E_{j}$ and also between $E_{j}$ and $P$ are substantial, we propose a trimming method for $w_{L_{i} E_{j}}$ and $w_{E_{\mathrm{j}} P}$ as follows: we set $w_{L_{\mathrm{i}} E_{\mathrm{j}}} \times w_{E_{\mathrm{j}} P}=0$, if $w_{L_{\mathrm{i}} E_{\mathrm{j}}}<\chi_{1, c_{1}}^{2}$ or $w_{E_{\mathrm{j}} P}<$ $\chi_{1, c_{2}}^{2}$. In the analysis below, we set

$$
c_{1}=c_{2}=(1-\sqrt{0.05}) \text {. }
$$

Then $w_{M P_{\mathrm{i}}}^{*}$ can be calculated as described above.

When only a subset of individuals have gene expression profiles available in a dataset, the $\mathrm{p}$ values can be derived from the full dataset, and weights can be calculated from the partial dataset. Then, the same weight-adjusting procedure can be implemented as described above. The R source code for implementing the proposed weighting procedure is available at: http://www.biostat.umn. edu/ yho/research.html.

\section{Simulation}

To mimic data from a GWA study, we constructed simulated data using the marginal distribution parameters of the genotype scores of the SNP markers, the gene expression measurements and 
Table 1. FWER of the conventional GWA analysis and 3 weightadjusting approaches in Scenarios 1 and 2

\begin{tabular}{lllll}
\hline & GWA & $W_{M P}$ & $W_{M P}^{T}$ & Li et al. [14] \\
\hline Scenario 1 & 0.053 & 0.055 & 0.053 & 0.051 \\
Scenario 2 & 0.046 & 0.048 & 0.047 & 0.078 \\
Scenario 3 & 0.051 & 0.051 & 0.051 & 0.059 \\
\hline
\end{tabular}

The results are from 10,000 simulation iterations. $W_{M P}$ : Weight adjustment approach without trimming. $W_{M P}^{T}$ : Weight adjustment approach with trimming.

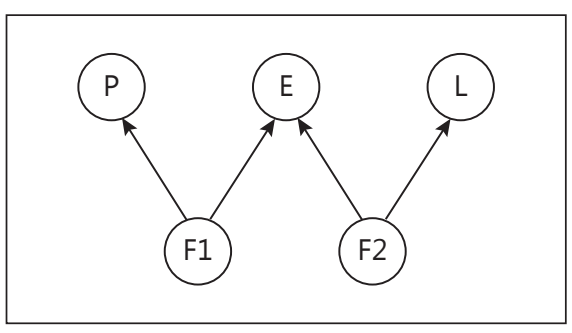

Fig. 1. A diagram illustrating simulation Scenario 3.

the phenotype obtained from the SLE study described in the previous section. We selected one SNP marker to be the true underlying $\mathrm{SNP}\left(S N P_{\text {true }}\right)$ and simulated a single gene expression level according to this model:

$$
E_{1}=\beta_{0 E}+\beta_{E} \times S N P_{\text {true }}+\varepsilon, \quad \varepsilon \sim N\left(0, \sigma_{E}^{2}\right) .
$$

In the described model, $\beta_{0 E}$ and $\sigma_{E}^{2}$ were obtained from the mean and the variance of a randomly selected gene expression. The value of the phenotypic outcome was simulated based on the model:

$$
P=\beta_{0 P}+\beta_{P} \times \frac{\left(E_{1}-\overline{E_{1}}\right)}{\sigma_{E_{1}}}+\varepsilon, \quad \varepsilon \sim N\left(0, \sigma_{P}^{2}\right) .
$$

$\beta_{0 P}$ and $\sigma_{P}$ were determined by the mean and variance of the chemokine score in the SLE dataset. We used standardized $E_{1}$ in the above equation for the ease of interpreting $\beta_{P}$.

To investigate family-wise error control, we randomly selected another 9 non-phenotype-associated SNP markers and 1,000 gene expression measurements from the experimental data in the SLE study. Hence, the simulated dataset consisted of 10 SNP markers, 1,001 gene expression measurements, and 1 continuous phenotypic outcome.

\section{Scenario 1}

In the first simulation, we assumed $\beta_{E}=\beta_{P}=0$ to examine the type I error rate. The results are presented in table 1 . In table 1 , we recorded the fraction of times that a SNP was declared significant at $\alpha=0.05 / 10$ for 4 approaches: the conventional GWA analysis, the weight-adjusted approach with $W_{M P}^{T}$ and without $W_{M P}$ trimming, and Li et al.'s approach [14]. The overall FWERs from the
10,000 simulations were estimated by counting the percent of times when any significant results were reported. In table 1, we observed the estimated FWERs were close to 0.05 for all 4 approaches. In addition, we observed similar results when assuming $\beta_{E}=10$, and $\beta_{P}=0$.

\section{Scenario 2}

A second simulation was conducted, assuming $\beta_{E}=0$ and $\beta_{P}=$ 10. The results are shown in table 1 . According to the simulation results shown in table 1 , the FWER remained controlled at the 0.05 level for GWA, $W_{M P}$, and $W_{M P}^{T}$.

\section{Scenario 3}

In this simulation scenario, the underlying SNP marker $L$ was not associated with outcome $P$ and gene expression $E$. However, 2 unobserved latent variables (F1 and F2) were in association with the observed $L, E$ and $P$, as illustrated in figure 1. This latent model can account population stratification or admixture. In this simulation scenario, we were interested in examining whether the latent variables could create a spurious dependency for the proposed analyses. According to the result shown in table 1, the FWER remained controlled at the 0.05 level for all 4 approaches in this scenario.

\section{Scenario 4}

To assess the power of the proposed weighting approaches, we conducted simulations assuming

$$
\frac{\beta_{E}}{\sigma_{E}}=\frac{\beta_{P}}{\sigma_{P}}=0.5 .
$$

The results are shown in table 2 . They suggested a substantial power gain by the weighting approaches, when compared to the conventional GWA analysis. By incorporating gene expression information in the moderate effect size setting

$$
\frac{\beta_{E}}{\sigma_{E}}=\frac{\beta_{P}}{\sigma_{P}}=0.5,
$$

power increased by $56 \%$ (from 42.5 to $66.1 \%$ ), when $W_{M P}^{T}$ was compared to the standard GWA analysis. Furthermore, the results also indicated that Li et al.'s approach [14] had a smaller power gain than our proposed weighting approaches due to the fact that Li et al.'s weight does not incorporate information of association between gene expression and phenotype.

\section{Scenario 5}

$$
\begin{aligned}
& S N P_{\text {true }} \rightarrow E_{1} \rightarrow P \\
& S N P_{\text {add }} \rightarrow E_{\text {add }}
\end{aligned}
$$

In this scenario, we assumed

$$
\frac{\beta_{E}}{\sigma_{E}}=\frac{\beta_{P}}{\sigma_{P}}=0.5 ;
$$

in addition, we assumed another SNP maker $\left(S N P_{a d d}\right)$ had an effect on the gene expression with effect size

$$
\frac{\beta_{\text {Eadd }}}{\sigma_{\text {Eadd }}}=2,
$$

but this gene expression was not associated with a phenotype as illustrated by the diagram below. The results from this simulation 
Table 2. Power of the conventional GWA analysis and 3 weightadjusting approaches in Scenarios 3, 4, and 5

\begin{tabular}{lllll}
\hline & GWA & $W_{M P}$ & $W_{M P}^{T}$ & Li et al. [14] \\
\hline Scenario 4 & 0.425 & 0.653 & 0.661 & 0.602 \\
Scenario 5 & 0.421 & 0.617 & 0.640 & 0.184 \\
Scenario 6 & 0.419 & 0.594 & 0.601 & 0.474 \\
Scenario 7 & 0.810 & 0.736 & 0.418 & 0.736 \\
Scenario 8 & 0.795 & 0.814 & 0.433 & 0.861 \\
\hline
\end{tabular}

The results are from 10,000 simulation iterations. $W_{M P}$ : Weight adjustment approach without trimming. $W_{M P}^{T}$ : Weight adjustment approach with trimming.

scenario are shown in table 2 . In table $2, W_{M P}$ and $W_{M P}^{T}$ demonstrated considerable power gains over the conventional GWA analysis. However, Li et al.'s weighting approach [14] suffered a dramatic power loss compared to the GWA approach in this scenario. Their weighting approach only accounted for the association between SNP and gene expression, which resulted in incorrectly assigning higher weights to $S N P_{a d d}$.

Scenario 6

In this scenario, we assumed

$$
\frac{\beta_{E}}{\sigma_{E}}=\frac{\beta_{P}}{\sigma_{P}}=0.5 ;
$$

only a subset of all individuals had expression measurements available, but more samples had SNP genotype and phenotype information. In this simulation, we assumed that only $1 / 3$ of all individuals had expression profiles available. The proposed weighting approach still demonstrated a considerable power gain (with an increase in power from $41.9 \%$ to $60.1 \%$ ) compared to the conventional GWA analysis; however, the power gain was less prominent than that of Scenario 4, when the expression profiles for the whole study population were available.

\section{Scenario 7}

This scenario assumes a reactive model [26] as illustrated by the diagram below. We assumed a SNP marker $L$ had an effect on outcome $P$, and the alteration of gene expression $E$ was the result of a change in outcome $P$. We assumed

$$
\frac{\beta_{P}}{\sigma_{P}}=\frac{\beta_{E}}{\sigma_{E}}=0.5
$$

in the simulation. The results shown in table 2 indicated that all 3 weight-adjusting approaches ( $W_{M P}, W_{M P}^{T}$, and Li et al.'s approach) assigned lower weights for SNP markers with gene expression in a reactive model compared to the original GWA p value. In the proposed weighting approaches, the model constructed for calculating $w_{L E}$ (equ. 1 ) adjusted for outcome $P$. As a result, $w_{L E}$ was less likely to be large and more likely be reduced to 0 in the trimming approach. Hence, we observed that $W_{M P}^{T}$ assigned the lowest weights for the reactive model.

$$
L \rightarrow P \rightarrow E \text {. }
$$

\section{Scenario 8}

This scenario is a modification from Scenario 5, where an independence model was assumed as illustrated in the diagram below. A SNP marker $L_{1}$ had an effect on outcome $P$ and also on gene expression $E_{1}$. But $P$ and $E_{1}$ were independent. We assumed

$$
\frac{\beta_{P}}{\sigma_{P}}=\frac{\beta_{E_{1}}}{\sigma_{E_{1}}}=0.3 \text { and } \frac{\beta_{E_{2}}}{\sigma_{E_{2}}}=2
$$

in the simulation. The results from this simulation scenario, shown in table 2, indicated that $W_{M P}^{T}$ assigned the lowest weights for SNP markers that arose from an independent model due to small $w_{E P}$ values. On the other hand, according to the simulation results, $\mathrm{Li}$ et al.'s approach [14] undesirably assigned larger weights for SNP markers that were in the independent model.

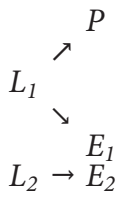

In summary, the proposed weighting procedures control the FWER at the nominal level when $L$ is not associated with $E, E$ is not associated with $P$, or both. Our proposed methods aimed to find genes that undergo a $L \rightarrow E \rightarrow P$ mechanism, where gene expression is in the middle of the pathway. Hence, our methods exhibited the largest power gain in identifying genes described in Scenario 4.

\section{Data Analysis Results}

\section{Results}

In the SLE dataset described in Section 2, the obtained chemokine score is considered a biomarker for lupus activity for SLE patients. To demonstrate the implementation of the proposed approach, we applied the chemokine score as the primary outcome and performed weight adjustment analyses for SNP loci with an allele frequency $>0.1$. The simulation results suggested that the trimming approach could allocate more weights on relevant SNP loci. Therefore, we applied $W_{M P}^{T}$ with trimming in the following analysis.

Table 3 lists the 13 SNPs with GWA p values $<10^{-5}$. In addition to the GWA p values indicated in the third column of table 3 , weights and weight-adjusted $\mathrm{p}$ values are also presented. In the last two columns of table 3, we recorded the annotation of SNP markers, and the gene expression that reported the maximum weight for the SNP. The weights obtained from the proposed $W_{M P}^{T}$ and Li et al.'s approach [14] have similar trends for 10 out of 16 top SNP markers shown in this table. Six SNP markers (rs2810693, rs11247300, rs931481, rs6536732, rs4892151, and rs122778761) were downweighted ( $w_{M P}^{*}$ $<1$ ) in our approach, while having $w>1$ using Li et al.'s
98

Hum Hered 2014;78:94-103 DOI: $10.1159 / 000362837$
Ho/Baechler/Ortmann/Behrens/

Graham/Bhangale/Pan 
Table 3. SNP loci with p value $<10^{-5}$ according to the GWA analysis or the weight adjustment procedure

\begin{tabular}{|c|c|c|c|c|c|c|c|c|}
\hline & SNP & Chr & $\begin{array}{l}\text { GWA } \\
\text { p value }\end{array}$ & $W_{M P}^{T}$ & $\begin{array}{l}\text { Li et al. } \\
{[14]}\end{array}$ & $\begin{array}{l}\text { Adjusted } \\
\text { p value }\end{array}$ & SNP annotation & Expression \\
\hline 1 & rs17415112 & 10 & $1.20 \mathrm{e}-07$ & 1.28 & 1.10 & $9.37 \mathrm{e}-08$ & EXOSC1 & LOC441019 \\
\hline 2 & rs7744944 & 6 & $1.48 \mathrm{e}-07$ & 1.38 & 1.37 & $1.07 \mathrm{e}-07$ & & SP140 \\
\hline 3 & rs6536732 & 4 & $5.57 \mathrm{e}-07$ & 0.98 & 1.54 & $5.69 \mathrm{e}-07$ & & RMI2 \\
\hline 4 & rs17218497 & 4 & $9.89 \mathrm{e}-07$ & 1.04 & 1.06 & $9.53 e-07$ & & CARD17 \\
\hline 5 & rs931481 & 4 & $2.96 \mathrm{e}-06$ & 0.71 & 1.11 & $4.18 \mathrm{e}-06$ & & \\
\hline 6 & rs4892151 & 18 & $4.30 \mathrm{e}-06$ & 0.74 & 1.03 & $5.79 \mathrm{e}-06$ & & CARD17 \\
\hline 7 & rs767528 & 20 & $4.34 \mathrm{e}-06$ & 1.89 & 1.01 & $2.29 \mathrm{e}-06$ & & IFI35 \\
\hline 8 & rs12778761 & 10 & $4.49 \mathrm{e}-06$ & 0.58 & 1.01 & $7.79 \mathrm{e}-06$ & ARHGAP19-SLIT1 & TNFSF10 \\
\hline 9 & rs11247300 & 15 & $5.53 e-06$ & 0.51 & 1.44 & $1.07 \mathrm{e}-05$ & PCSK6 & LGALS9 \\
\hline 10 & rs306254 & 5 & $5.83 \mathrm{e}-06$ & 0.68 & 0.90 & $8.54 \mathrm{e}-06$ & & SP140 \\
\hline 11 & rs2810693 & 13 & $6.90 \mathrm{e}-06$ & 0.63 & 1.17 & $1.10 \mathrm{e}-05$ & & GBP5 \\
\hline 12 & rs173577 & 5 & $6.99 \mathrm{e}-06$ & 0.57 & 0.92 & $1.23 \mathrm{e}-05$ & & RNASE2 \\
\hline$\overline{13}$ & rs4919096 & 10 & $8.09 \mathrm{e}-06$ & 0.66 & 0.80 & $1.23 \mathrm{e}-05$ & & TIMM10 \\
\hline 14 & rs596346 & 11 & $1.22 \mathrm{e}-05$ & 1.54 & 1.30 & $7.95 e-06$ & ВC041900 & FAM212B \\
\hline 15 & rs624676 & 11 & $1.40 \mathrm{e}-05$ & 1.46 & 1.39 & $9.55 \mathrm{e}-06$ & ВС041900 & FAM212B \\
\hline 16 & rs514604 & 11 & $1.40 \mathrm{e}-05$ & 1.46 & 1.39 & $9.55 e-06$ & BC041900 & FAM212B \\
\hline
\end{tabular}

Chr $=$ Chromosome. Adjusted $\mathrm{p}$ value is calculated as $\frac{\text { GWAS p value }}{w_{M P}^{*}}$.

approach [14]. One possible explanation is that these SNP markers might undergo the independent model.

The top SNP hit (rs17415112) is located within the EXOSC1 gene. EXOSC1 encodes a core component of the exosome and may be involved in Ig class switch recombination and degradation of mRNA transcripts for histone proteins, which are implicated in SLE [27]. Our results suggest a possible interaction of EXOSC1 and LOC441019, a locus related to immune system, cytokine, and IFN- $\gamma$ signaling. In addition, several transcripts reported in table 3 are involved in the immune response, inflammation, and cytokine pathways that are known to be associated with active SLE [28]. These include inflammatory caspase-related CARD17 [29], IFN-inducible IFI35, apoptosis-inducing ligand TNFSF10 [30], and galectin LGALS9 [31]. Furthermore, according to table 3, we noticed that there were several SNP markers at the top of the list having lowered $\mathrm{p}$ values after weight adjustment. Using the threshold of $10^{-5}, 3$ additional SNPs (rs596346, rs624676, and rs514604) became significant. These 3 SNP markers are in linkage disequilibrium and mapped to a noncoding mRNA transcript BC041900. This region is about $500-\mathrm{kb}$ upstream of gene clusters on chromosome 11, where FAM181B, PRCP, SNORA70E, C11orf82, LOC100506233, AK311356, ANKRD42, and $R A B 30$ locate.

Using Gene Expression to Improve the Power of GWA
To investigate SNP loci with moderate $\mathrm{p}$ values but large weights, we ranked SNP loci with unadjusted $\mathrm{p}$ value $<10^{-3}$ and $w_{M P}^{*}>1$. The top 40 ranked loci are listed in table 4. The complete list of SNP loci with unadjusted $\mathrm{p}$ value $<10^{-3}$ and $w_{M P}^{*}>1$ is available in online supplementary table S1 (www.karger.com/doi/10.1159/000362837). Interestingly, in some cases, multiple SNPs on different chromosomes appear to interact with a single transcript. Among these transcripts are BST2, PARP12, SP140, TIMM10, UBQLNL, and XAF1. This suggests that distinct genetic variants may lead to an altered expression of a single gene and provide divergent ways to trigger elevated chemokine levels. In table 4, there are 11 SNP markers which were upweighted $\left(w_{M P}^{*}>1\right)$ using our approach but downweighted by Li et al.'s approach [14]. These differences in weights could be due to the mechanism described in Scenario 2.

\section{Functional Annotation}

Many of the transcripts identified in our study are known to be regulated by type I IFN [16]. Furthermore, several of the transcript abundances are also known to be altered in blood cells of patients with SLE compared to healthy controls [16]. Among those transcript abundances that are both altered in SLE and IFN inducible are IFI35, TNFSF10, XAF1, PARP12, BST2, ISG15, DDX58, 
Table 4. Top $40 W_{M P}^{*}$ ranked SNP loci with unadjusted $\mathrm{p}$ value $<10^{-3}$ and $W_{M P}^{*}>1$

\begin{tabular}{|c|c|c|c|c|c|c|c|c|c|}
\hline & SNP & $\mathrm{Chr}$ & $\begin{array}{l}\text { GWA } \\
\text { p value }\end{array}$ & $W_{M P}^{T}$ & Li et al. [14] & $\begin{array}{l}\text { Adjusted } \\
\text { p value }\end{array}$ & $\begin{array}{l}\text { GWA } \\
\text { rank }\end{array}$ & $\begin{array}{l}\text { SNP } \\
\text { annotation }\end{array}$ & Expression \\
\hline 1 & rs6495528 & 15 & $9.74 \mathrm{e}-04$ & 6.24 & 0.94 & $1.56 \mathrm{e}-04$ & 580 & KIAA1199 & XAF1 \\
\hline 3 & rs12374087 & 3 & $7.05 e-04$ & 3.55 & 0.96 & $1.99 \mathrm{e}-04$ & 421 & & TIMM10 \\
\hline 4 & rs12681426 & 8 & $4.66 \mathrm{e}-04$ & 3.30 & 1.00 & $1.41 \mathrm{e}-04$ & 299 & & PARP12 \\
\hline 7 & rs2086369 & 8 & $9.50 \mathrm{e}-04$ & 3.01 & 1.38 & $3.16 \mathrm{e}-04$ & 566 & NKAIN3 & BATF2 \\
\hline 8 & rs1021012 & 8 & $8.81 \mathrm{e}-04$ & 2.97 & 1.36 & $2.97 \mathrm{e}-04$ & 527 & NKAIN3 & BATF2 \\
\hline 9 & rs7015211 & 8 & $8.81 \mathrm{e}-04$ & 2.97 & 1.36 & $2.97 \mathrm{e}-04$ & 527 & NKAIN3 & BATF2 \\
\hline 10 & rs4721478 & 7 & $8.41 \mathrm{e}-04$ & 2.86 & 0.77 & $2.94 \mathrm{e}-04$ & 506 & ISPD & MDK \\
\hline 11 & rs4809388 & 20 & $4.94 \mathrm{e}-04$ & 2.83 & 1.60 & $1.74 \mathrm{e}-04$ & 313 & PRPF6 & SP140 \\
\hline 12 & rs2071570 & 17 & $2.61 \mathrm{e}-04$ & 2.83 & 0.96 & $9.22 \mathrm{e}-05$ & 178 & NR1D1 & PARP12 \\
\hline 17 & rs12986829 & 2 & $6.12 \mathrm{e}-05$ & 2.18 & 1.52 & $2.81 \mathrm{e}-05$ & 56 & & TIMM10 \\
\hline 18 & rs1400030 & 2 & $1.21 \mathrm{e}-04$ & 2.18 & 1.47 & $5.55 \mathrm{e}-05$ & 84 & & TIMM10 \\
\hline 19 & rs12536527 & 7 & $9.26 \mathrm{e}-04$ & 2.13 & 1.19 & $4.34 \mathrm{e}-04$ & 556 & SDK1 & GBP5 \\
\hline 20 & rs13426554 & 2 & $3.45 e-05$ & 2.12 & 1.39 & $1.63 \mathrm{e}-05$ & 38 & & DDX58 \\
\hline 21 & rs2345102 & 2 & $3.45 e-05$ & 2.12 & 1.39 & $1.63 e-05$ & 38 & & DDX58 \\
\hline 22 & rs11203933 & 8 & $2.73 e-04$ & 2.10 & 0.95 & $1.30 \mathrm{e}-04$ & 187 & & SP140 \\
\hline 23 & rs7912066 & 10 & $6.61 \mathrm{e}-04$ & 2.02 & 0.86 & $3.27 \mathrm{e}-04$ & 397 & CTNNA3 & TIMM10 \\
\hline 24 & rs 4402422 & 13 & $2.19 \mathrm{e}-04$ & 2.02 & 1.21 & $1.09 \mathrm{e}-04$ & 149 & & UBQLNL \\
\hline 25 & rs12329145 & 2 & $1.01 \mathrm{e}-04$ & 2.02 & 1.41 & $4.99 \mathrm{e}-05$ & 76 & & TIMM10 \\
\hline 26 & rs1041530 & 10 & $1.24 \mathrm{e}-04$ & 1.99 & 1.21 & $6.21 \mathrm{e}-05$ & 88 & & SP140 \\
\hline 27 & rs4739003 & 8 & $2.54 \mathrm{e}-04$ & 1.99 & 1.48 & $1.28 \mathrm{e}-04$ & 173 & NKAIN3 & UBQLNL \\
\hline 35 & rs12710667 & 2 & $6.26 \mathrm{e}-04$ & 1.88 & 0.79 & $3.34 \mathrm{e}-04$ & 378 & & PARP12 \\
\hline 36 & rs988667 & 11 & $2.47 e-04$ & 1.87 & 1.18 & $1.32 \mathrm{e}-04$ & 164 & DKK3 & LOC40075s \\
\hline 37 & rs 2161210 & 19 & $6.77 e-04$ & 1.86 & 1.23 & $3.65 \mathrm{e}-04$ & 404 & & MT2A \\
\hline 38 & rs647619 & 1 & $8.13 e-04$ & 1.84 & 1.35 & $4.42 \mathrm{e}-04$ & 481 & & TNFSF10 \\
\hline 39 & rs761523 & 14 & $3.86 e-04$ & 1.84 & 0.94 & $2.11 \mathrm{e}-04$ & 250 & & BST2 \\
\hline 40 & rs12882238 & 14 & $5.86 e-05$ & 1.82 & 0.97 & $3.22 \mathrm{e}-05$ & 52 & & XAF1 \\
\hline
\end{tabular}

Chr $=$ Chromosome. Adjusted $\mathrm{p}$ value is calculated as $\frac{\text { GWAS p value }}{w_{M P}^{*}}$.

HERC6, and MT2A. Only a few transcripts in our results (SP140, PSME2, and LGALS9) are IFN inducible but were not observed to be changed in our studies of SLE patients.
While the current findings require validation to confirm the association of these variants with gene expression and serum chemokine levels, several of the identified loci harbor genes with known functions related to the immune system. These include DKK3, which plays a role in 
peripheral CD8 T-cell tolerance [32]; transcription factor NR1D1, which regulates the production of inflammatory cytokines [33]; RGS9, which may have a role in chemokine-induced lymphocyte migration [34], and SDK1, which is associated with combined variable immunodeficiency [35]. In addition, several expressed genes listed in the ninth column of table 4 , which interact with the SNP variants described above, participate in cellular apoptosis, such as XAF1, UBQLNL, TIMM10, BST2, HERC6, ISG15, and PSME2 [36, 37].

\section{Discussion}

Through experimental data analysis, our primary focus was to demonstrate the implementation of the proposed weight adjustment approach. In the analysis of SLE GWA data, we identified 3 SNP loci with large weights that became significant after weight adjustment, as shown in table 3. Several genes listed in tables 3 and 4 have pivotal roles in immune functions. Our results identified several genetic interactions among immune responses and cellular apoptosis pathways and seem to suggest the importance of their interactions in active SLE symptoms. These SNP loci and the corresponding, associated gene expressions provided valuable information for further functional evaluations. A replication of these findings in other cohorts is necessary to demonstrate the biological significance of the additional loci identified by our method.

Yet, we also observed SNP loci with small p values that showed no evidence of gene expression association when analyzing SLE GWA data. This could be so because phenotype contribution mechanisms were not measured in the current study, such as through unmeasured gene expression transcripts, DNA structures, or other mechanisms. These SNPs will not be favored in the proposed weighting method, since they will be downweighted due to a lack of evidence of association with gene expression. These unmeasured mechanisms might explain the moderate differences in $\mathrm{p}$ values we observed in the SLE data analysis results described in Data Analysis Results. Hence in practice, we suggest to pursue both (1) SNPs with tiny GWA p values without weighting and (2) SNPs with small weight-adjusted p values. The proposed weighting approach assists researchers to prioritize GWA SNP findings by integrating available genomic information.

In this paper, we demonstrated our method in a paired gene expression and GWA study data from the same co- hort. To date, relatively few published studies have utilized gene expression data from the same patients studied by GWA study [38-42]. However, as appreciation grows for the power of eQTL analysis, efforts such as the Genotype-Tissue Expression project will come to fruition [43] and methods such as ours will facilitate the analysis of such datasets. Therefore, we believe the collection of RNA from GWA study participants for expression profiling will be useful. In addition, if eQTL (SNP, gene expression) data, and gene expression-phenotype data are available from two different sets of cohorts, instead of paired gene expression and GWA study data from the same cohort, our proposed approaches can be easily modified and applied. Our proposal provides a formalized procedure to incorporate additional genomic information into GWA analyses. In additional to gene expression measurements as demonstrated in this paper, with the advent of a wealth of genetic data generated through high-throughput technologies, the proposed method is extendable to integrate other sources of information such as DNA methylation status, transcription regulation, and protein abundance.

The weighted hypothesis testing concept was first introduced by Holm [22] and, since then, theoretical developments have been advanced to form the basis for $p$ value weighting in order to increase power while controlling FWER in a multiple hypothesis testing setting. Roeder and Wasserman [25] provided an applicable theory for constructing weights which control FWER at the nominal level. Roeder et al. [24] applied p value weighting procedures to GWA analysis and demonstrated power gain compared to conventional analysis.

In the spirit of integrating genomic information from multiple sources for power gain, we proposed novel weighting procedures based on the theory by Roeder and Wasserman [25] to incorporate gene expression into GWA analyses. Our simulation results confirmed that the proposed weighting procedure dramatically improved the statistical power of GWA studies, while controlling FWER at the nominal level, when gene expression is in the middle of the etiological pathway. Using this mechanism, our methods demonstrated greater power gain compared to that of Li et al. [14]. It also provides ways to draw valuable information from massive data to assist functional interpretations of GWA signals. 


\section{Acknowledgement}

Y.-Y.H. is supported by NIH grants P30 CA77598, P50CA101955, UL1TR000114, and U54MD008620. W.P. is supported by NIH grants R01GM081535, R01HL65462, and R01HL105397.

\section{Disclosure Statement}

The data collection was supported by the Alliance for Lupus Research and the Arthritis Foundation. W.O., T.W.B., R.R.G., and T.R.B. are employees of Genentech, Inc.

\section{References}

$\checkmark 1$ Hindorff LA, Sethupathy P, Junkins HA, Ramos EM, Mehta JP, Collins FS, Manolio TA: Potential etiologic and functional implications of genome-wide association loci for human diseases and traits. Proc Natl Acad Sci USA 2009;106:9362-9367.

2 Manolio TA, Collins FS, Cox NJ, et al: Finding the missing heritability of complex diseases. Nature 2009;461:747-753.

-3 Conlon EM, Liu XS, Lieb JD, Liu JS: Integrating regulatory motif discovery and genomewide expression analysis. Proc Natl Acad Sci USA 2003;100:3339-3344.

- 4 Wu Z, Sahin O, Shen Z, Liu P, Miller WG, Zhang Q: Multi-omics approaches to deciphering a hypervirulent strain of Campylobacter jejuni. Genome Biol Evol 2013;5:22172230.

5 Nicolae DL, Gamazon E, Zhang W, Duan S, Dolan ME, Cox NJ: Trait-associated SNPs are more likely to be eQTLs: annotation to enhance discovery from GWAS. PLoS Genet 2010;6:e1000888.

6 Li S, Lu Q, Cui Y: A systems biology approach for identifying novel pathway regulators in eQTL mapping. J Biopharm Stat 2010;20: 373-400.

7 Li S, Williams BL, Cui Y: A combined p-value approach to infer pathway regulations in eQTL mapping. Stat Interface 2011;4:389402.

8 Cusanovich DA, Billstrand C, Zhou X, Chavarria C, De Leon S, Michelini K, Pai AA, Ober C, Gilad Y: The combination of a genome-wide association study of lymphocyte count and analysis of gene expression data reveals novel asthma candidate genes. Hum Mol Genet 2012;21:2111-2123.

9 Yang C, Wang L, Zhang S, Zhao H: Accounting for non-genetic factors by low-rank representation and sparse regression for eQTL mapping. Bioinformatics 2013;29:1026-1034.

10 Kendziorski CM, Chen M, Yuan M, Lan H, Attie AD: Statistical methods for expression quantitative trait loci (eQTL) mapping. Biometrics 2006;62:19-27.

11 Sun W: A statistical framework for eQTL mapping using RNA-seq data. Biometrics 2012;68:1-11.
Chen Y, Zhu J, Lum PY, Yang X, Pinto S, MacNeil DJ, Zhang C, Lamb J, Edwards S, Sieberts SK, Leonardson A, Castellini LW, Wang S, Champy MF, Zhang B, Emilsson V, Doss S, Ghazalpour A, Horvath S, Drake TA, Lusis AJ, Schadt EE: Variations in DNA elucidate molecular networks that cause disease. $\mathrm{Na}-$ ture 2008;452:429-435.

13 Genovese CR, Roeder K, Wasserman L: False discovery control with $\mathrm{p}$ value weighting. Biometrika 2006;93:509-524.

14 Li L, Kabesch M, Bouzigon E, Demenais F, Farrall M, Moffatt MF, Lin X, Liang L: Using eQTL weights to improve power for genomewide association studies: a genetic study of childhood asthma. Front Genet 2013;4:103.

15 Bronson PG, Chaivorapol C, Ortmann W, Behrens TW, Graham RR: The genetics of type I interferon in systemic lupus erythematosus. Curr Opin Immunol 2012;24:530-537.

16 Baechler EC, Batliwalla FM, Karypis G, Gaffney PM, Ortmann WA, Espe KJ, Shark KB, Grande WJ, Hughes KM, Kapur V, Gregersen PK, Behrens TW: Interferon-inducible gene expression signature in peripheral blood cells of patients with severe lupus. Proc Natl Acad Sci USA 2003;100:2610-2615.

17 Bennett L, Palucka AK, Arce E, Cantrell V, Borvak J, Banchereau J, Pascual V: Interferon and granulopoiesis signatures in systemic lupus erythematosus blood. J Exp Med 2003; 197:711-723.

18 Crow MK, Kirou KA, Wohlgemuth J: Microarray analysis of interferon-regulated genes in SLE. Autoimmunity 2003;36:481-490.

19 Bauer JW, Baechler EC, Petri M, Batliwalla FM, Crawford D, Ortmann WA, Espe KJ, Li W, Patel DD, Gregersen PK, Behrens TW: Elevated serum levels of interferon-regulated chemokines are biomarkers for active human systemic lupus erythematosus. PLoS Med 2006;3:e491.

20 Bauer JW, Petri M, Batliwalla FM, Koeuth T, Wilson J, Slattery C, Panoskaltsis-Mortari A, Gregersen PK, Behrens TW, Baechler EC: Interferon-regulated chemokines as biomarkers of systemic lupus erythematosus disease activity: a validation study. Arthritis Rheum 2009;60:3098-3107.

21 Fangtham M, Petri M: 2013 update: Hopkins lupus cohort. Curr Rheumatol Rep 2013;15: 360-366.
22 Holm S: A simple sequentially rejective multiple test procedure. Scand J Stat 1979;6:6570.

23 Roeder K, Devlin B, Wasserman L: Improving power in genome-wide association studies: weights tip the scale. Genet Epidemiol 2007; 31:741-747.

24 Roeder K, Bacanu SA, Wasserman L, Devlin $\mathrm{B}$ : Using linkage genome scans to improve power of association in genome scans. Am J Hum Genet 2006;78:243-252.

25 Roeder K, Wasserman L: Genome-wide significance levels and weighted hypothesis testing. Stat Sci 2009;24:398-413.

26 Schadt EE, Lamb J, Yang X, Zhu J, Edwards S, Guhathakurta D, Sieberts SK, Monks S, Reitman M, Zhang C, Lum PY, Leonardson A, Thieringer R, Metzger JM, Yang L, Castle J, Zhu H, Kash SF, Drake TA, Sachs A, Lusis AJ: An integrative genomics approach to infer causal associations between gene expression and disease. Nat Genet 2005;37:710-717.

27 Fishbein E, Alarcon-Segovia D, Vega JM: Antibodies to histones in systemic lupus erythematosus. Clin Exp Immunol 1979;36:145150.

28 Rahman A, Isenberg DA: Systemic lupus erythematosus. N Engl J Med 2008;358:929-939.

29 Lamkanfi M, Denecker G, Kalai M, D'hondt K, Meeus A, Declercq W, Saelens X, Vandenabeele P: INCA, a novel human caspase recruitment domain protein that inhibits interleukin-1 $\beta$ generation. J Biol Chem 2004; 279:51729-51738.

- 30 Pitti RM, Marsters SA, Ruppert S, Donahue CJ, Moore A, Ashkenazi A: Induction of apoptosis by apo-2 ligand, a new member of the tumor necrosis factor cytokine family. J Biol Chem 1996;271:12687-12690.

31 Zhu C, Anderson AC, Schubart A, Xiong H, Imitola J, Khoury SJ, Zheng XX, Strom TB, Kuchroo VK: The Tim-3 ligand galectin-9 negatively regulates $\mathrm{T}$ helper type 1 immunity. Nat Immunol 2005;6:1245-1252.

32 Papatriantafyllou M, Moldenhauer G, Ludwig J, Tafuri A, Garbi N, Hollmann G, Küblbeck G, Klevenz A, Schmitt S, Pougialis G, Niehrs C, Gröne HJ, Hämmerling GJ, Arnold B, Oelert T: Dickkopf-3, an immune modulator in peripheral CD8 T-cell tolerance. Proc Natl Acad Sci USA 2012;109:16311636. 
33 Gibbs JE, Blaikley J, Beesley S, Matthews L, Simpson KD, Boyce SH, Farrow SN, Else KJ, Singh D, Ray DW, Loudon ASI: The nuclear receptor REV-ERB $\alpha$ mediates circadian regulation of innate immunity through selective regulation of inflammatory cytokines. Proc Natl Acad Sci USA 2012;109:582-587.

34 Agenès F, Bosco N, Mascarell L, Fritah S, Ceredig R: Differential expression of regulator of G-protein signalling transcripts and in vivo migration of $\mathrm{CD} 4+$ naïve and regulatory T cells. Immunology 2005; 115:179188.

35 Keller MD, Jyonouchi S: Chipping away at a mountain: Genomic studies in common variable immunodeficiency. Autoimmun Rev 2012;12:687-689.
6 Straszewski-Chavez SL, Visintin IP, Karassina N, Los G, Liston P, Halaban R, Fadiel A, Mor G: XAF1 mediates tumor necrosis factora-induced apoptosis and X-linked inhibitor of apoptosis cleavage by acting through the mitochondrial pathway. J Biol Chem 2007; 282:13059-13072.

37 Sijts EJAM, Kloetzel PM: The role of the proteasome in the generation of MHC class I ligands and immune responses. Cell Mol Life Sci 2011;68:1491-1502.

38 Schadt EE, Molony C, Chudin E, et al: Mapping the genetic architecture of gene expression in human liver. PLoS Biol 2008;6:e107.

39 Emilsson V, Thorleifsson G, Zhang B, et al: Genetics of gene expression and its effect on disease. Nature 2008;452:423-428.
40 Borel C, Deutsch S, Letourneau A, Migliavacca E, Montgomery SB, Dimas AS, Vejnar CE, Attar H, Gagnebin M, Gehrig C, Falconnet E, Dupré Y, Dermitzakis ET, Antonarakis SE: Identification of cis-and trans-regulatory variation modulating microrna expression levels in human fibroblasts. Genome Res 2011;21:68-73.

41 Hao K, Bossé Y, Nickle DC, et al: Lung eQTLs to help reveal the molecular underpinnings of asthma. PLoS Genet 2012;8:e1003029.

42 Almlöf JC, Lundmark P, Lundmark A, et al: Powerful identification of Cis-regulatory SNPs in human primary monocytes using allele-specific gene expression. PLoS One 2012; 7:e52260.

43 Lonsdale J, Thomas J, Salvatore M, et al: The Genotype-Tissue Expression (GTEx) project. Nature Genetics 2013;45:580-585.

\section{Erratum}

In the article 'Delta-centralization fails to control for population stratification in genetic association studies' by Dadd et al. [Hum Hered 2010;69:285-294] the following errors appeared:

1) Reference 10 should refer to: Devlin B, Roeder K and Wasserman L: Genomic control, a new approach to genetic-based association studies. Theor Popul Biol 2001;60:155166.

2) On page 288, the equation for the bias parameter $\delta_{T}$ should be:

$$
\delta_{T}=\sum_{j=1}^{K} p_{T j} f_{j} / s
$$

Also the term ' $s$ ' needs to be defined as: 'where $s$ is an estimate of the standard deviation of the difference in allele frequency between cases and controls.'

3) On page 288 , the equation for $\operatorname{Var}\left[\delta_{T}\right]$ should be:

$$
\operatorname{Var}\left[\delta_{i}\right]=F_{S T} p_{i}\left(1-p_{i}\right) \sum_{j=1}^{K} f_{j}^{2} / s^{2}
$$

4) On page 288 , the equation for $\eta^{2}$ should be:

$$
\eta^{2}=n F_{S T} \sum_{j=1}^{K} f_{j}^{2}
$$

5) On page 288, the expression

$$
\sum_{j=1}^{K} f_{j}
$$

which appears half way down the right hand side should be:

$$
\sum_{j=1}^{K} f_{j}^{2}
$$

Using Gene Expression to Improve the Power of GWA 